\title{
A note on the horseshoe confinement model: The Poynting-Robertson effect
}

\author{
S. M. Giuliatti Winter, O. C. Winter, and A. H. F. Guimarães \\ Grupo de Dinâmica Orbital \& Planetologia, UNESP, CP 205, Guaratinguetá, CEP 12500-000, SP, Brazil \\ Received 22 October 2002 / Accepted 31 December 2003

\begin{abstract}
In the present work we numerically simulated the motion of particles coorbital to a small satellite under the Poynting-Robertson light drag effect in order to verify the symmetry suggested by Dermott et al. $(1979,1980)$ on their ring confinement model. The results reveal a more complex scenario, especially for very small particles (micrometer sizes), which present chaotic motion. Despite the complexity of the trajectories the particles remain confined inside the coorbital region. However, the dissipative force caused by the solar radiation also includes the radiation pressure component which can change this configuration. Our results show that the inclusion of the radiation pressure, which is not present in the original confinement model, can destroy the configuration in a time much shorter than the survival time predicted for a dust particle in a horseshoe orbit with a satellite.
\end{abstract}

Key words. planets: rings - solar system: general

\section{Introduction}

Goldreich \& Tremaine (1979) have pointed out that the effects of inter-particle collisions and Poynting-Robertson light drag on unconstrained rings lead to radial spreading, disrupting a ring in a time scale far shorter than the age of the solar system. This would mean that the rings were formed recently or are being continuously replenished. To avoid such a time scale constraint two confinement models have been proposed. The concept of shepherding satellites was proposed by Goldreich \& Tremaine (1979). This model consists of two small satellites, one interior and other exterior to the ring, that would prevent radial spreading by the gravitational torque on the ring in a direction away from the perturbing satellites. The ring edges would be located at the balance of these two torques. This formalism assumes both satellites and ring particles to be on circular orbits. The discovery of the satellites Cordelia and Ophelia shepherding the Uranian $\epsilon$ ring confirmed this model (Porco \& Goldreich 1987). Murray \& Thompson (1990) proposed that unseen moonlets could be the shepherd satellites of the other narrow rings of Uranus. The other mechanism for confining narrow rings, proposed by Dermott et al. (1979, 1980), consists of one small satellite embedded within the ring. The ring particles would be in stable horseshoe orbits about the classical Lagrangian points $L_{3}, L_{4}$ and $L_{5}$. An example of this mechanism was found in the Encke Gap central ringlet where the satellite Pan is claimed to be responsible for confining the ring

Send offprint requests to: S. M. Giuliatti Winter, e-mail: silvia@feg.unesp.br material (Showalter 1991). In this model, the mass ratio between the satellite and the planet has to be $\mu \leq 10^{-9}$. For very narrow rings the variation in the semi major axis of the particle before and after the encounter with the satellite is almost exactly equal and opposite (Dermott \& Murray 1981). Dermott et al. (1980), Dermott (1984) and Murray \& Dermott (1999) argue that the Poynting-Robertson light drag (PRdrag) has little influence on the encounter with the satellite, and the PRdrag forces acting on the particle were the same in both halves of the horseshoe path. Then, the orbital decay of the particle achieved in one half of the horseshoe path would in effect be cancelled by that achieved in the other half. A schematic diagram of this model, as proposed by Dermott (1984), can be seen in Fig. 1. The path of a horseshoe particle as a function of its angular position, in the rotating reference frame, is shown by the dashed line, while the solid line shows the change in this path due to the effects of the PRdrag force. The satellite is located at $r=1$ and $\theta=0^{\circ}$. In this analysis the authors decoupled the effects of PRdrag on the particle from the gravitational perturbation by the satellite. In the present work we numerically simulated the coupled effect on this dynamical system. The goal of this paper is to verify the validity of the model proposed by Dermott et al. (1980). This paper also takes a step further by including the radiation pressure in this confinement model. Despite the fact that the radiation pressure component causes a secular variation in the eccentricity of the particle (Burns et al. 1979), this effect can alter the dynamical system evolution. The paper is organized as follows: in Sect. 2 we derive the equations of motion for the particle in a horseshoe orbit with a satellite in the 


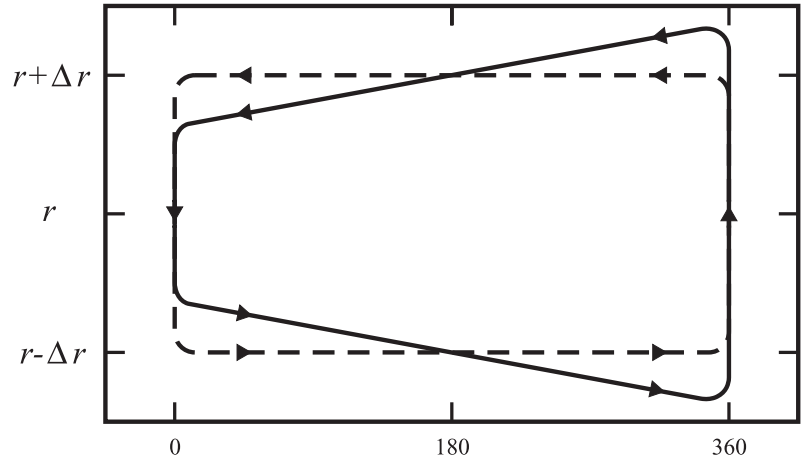

Fig. 1. A diagram showing the paths of a horseshoe particle under the influence of the PRDrag (solid line) and without taking into account this perturbation (dashed line).

presence of solar radiation, in Sect. 3 the numerical simulations are presented and in the last section the results are analysed.

\section{Dynamical system}

Schuerman (1979) analysed the stability and location of the Lagrangian points in the planar circular restricted three body problem when some dissipative forces are taken into account. His results show that the points $L_{4}$ and $L_{5}$ are linearly unstable under the effects of PRdrag for particles in orbits around the Sun. Nevertheless, his analysis cannot be applied either to large-amplitude orbits, such as the horseshoe orbits, or to particles in orbit around a planet. In the present work we are interested in the effect of PRdrag on the orbital evolution of a particle in horseshoe orbit with a satellite around a planet, and also in the effects of the radiation pressure component (RP). The dynamical system considered here is within the framework of the restricted three-body problem, planet-satellite-ring particle, with a time-dependent additional force, whose direction will change regularly over the synodic period of the satellite. The vectorial expression for the PRdrag and RP on a particle around a planet is given by (Mignard 1984)

$\boldsymbol{F}=\beta\left\{\frac{\boldsymbol{r}_{\mathrm{sp}}}{r_{\mathrm{sp}}}\left[1-\frac{\boldsymbol{r}_{\mathrm{sp}}}{r_{\mathrm{sp}}}\left(\frac{\boldsymbol{v}_{\mathrm{p}}}{c}+\frac{\boldsymbol{v}}{c}\right)\right]-\left(\frac{\boldsymbol{v}_{\mathrm{p}}}{c}+\frac{\boldsymbol{v}}{c}\right)\right\}$

where $c$ is the speed of light, $\boldsymbol{v}$ is the velocity vector of the particle relative to the planet, $\boldsymbol{r}_{\mathrm{sp}}$ is the Sun-planet position vector, $\boldsymbol{v}_{\mathrm{p}}$ is the velocity vector of the planet relative to the Sun and $r_{\mathrm{sp}}=\left|\boldsymbol{r}_{\mathrm{sp}}\right|$. The first term corresponds to the RP component and the other terms to the PRdrag component. For spherical particles which obey geometrical optics, the value of $\beta$ is given by (Burns et al. 1979)

$\beta=5.7 \times 10^{-5} \frac{Q_{\mathrm{pr}}}{\rho s}$

where $s$ and $\rho$ are the radius and the density of a spherical particle in CGS units and $Q_{\mathrm{pr}}$ is a constant value which depends on the optical properties of the grain.

The equations of motion for a ring particle in a planar orbit around the planet (with mass $m_{1}$ ), in a barycentric frame rotating with the same angular velocity of the satellite (with mass $m_{2}$ ), under the effects of a dissipative force, are given by (Murray \& Dermott 1999)

$\ddot{x}-2 \dot{y}=\frac{\partial U}{\partial x}+F_{x}$
$\ddot{y}+2 \dot{x}=\frac{\partial U}{\partial y}+F_{y}$

where $U=U(x, y)$ is given by

$U=\frac{\left(x^{2}+y^{2}\right)}{2}+\frac{\mu_{1}}{r_{1}}+\frac{\mu_{2}}{r_{2}}$

where $(x, y)$ and $(\dot{x}, \dot{y})$ are the coordinates of the position and the velocity of the particle respectively, $\mu_{2}=\left(m_{2} /\left(m_{1}+m_{2}\right)\right)$ and $\mu_{1}=1-\mu_{2}$. The distance between the satellite and the planet is taken to equal one, consequently the mean motion of the satellite around the planet is equal one. The values of $r_{1}$ and $r_{2}$ are

$$
r_{1}^{2}=\left(x+\mu_{2}\right)^{2}+y^{2}, \quad r_{2}^{2}=\left(x-\mu_{1}\right)^{2}+y^{2}
$$

and $F_{x}$ and $F_{y}$, the components of the RP and PRdrag derived from Eq. (1) are

$$
\begin{aligned}
F_{x}= & -\frac{\beta G M_{\text {sun }}}{d^{2}}\left\{\cos \left(n_{\text {sun }} t\right)+(\dot{x}-y) \cos ^{2}\left(n_{\text {sun }} t\right)\right. \\
& \left.+(\dot{y}+x) \cos \left(n_{\text {sun }} t\right) \sin \left(n_{\text {sun }} t\right)\right\} \\
F_{y}= & -\frac{\beta G M_{\text {sun }}}{d^{2}}\left\{\sin \left(n_{\text {sun }} t\right)+(\dot{y}+x) \sin ^{2}\left(n_{\text {sun }} t\right)\right. \\
& \left.+(\dot{x}-y) \cos \left(n_{\text {sun }} t\right) \sin \left(n_{\text {sun }} t\right)\right\}
\end{aligned}
$$

where $M_{\text {sun }}$ and $n_{\text {sun }}$ are the mass and the mean motion of the Sun in the rotating coordinate system respectively, $d$ is the distance between the planet and the Sun and $G$ is the gravitational constant. In this model the planet is in circular orbit around the Sun.

\section{Numerical simulations}

In order to analyse the effects of the RP and PRdrag on a small particle initially in a horseshoe orbit with a satellite, we have numerically simulated the system adopting the equations of motion (Eq. (3)). We have used the Gauss-Radau numerical integrator (Everhard 1985).

The satellite is in circular orbit around the planet and the distance from the satellite to the planet is taken to be equal to the semi-major axis of the saturnian satellite Pan (Murray \& Dermott 1999).

The initial conditions taken correspond to those particles which perform a horseshoe orbit in the restricted three body problem when the dissipative force is not considered. The particle is initially located inside the horseshoe region and its initial angular position is $\theta=180^{\circ}$ from the satellite. The width of the horseshoe region is derived, for each satellite mass, from the theory developed by Dermott \& Murray (1981).

Dermott et al. (1979) proposed that the horseshoe confinement model is verified when the satellite mass is such that $\mu_{2} \leq 10^{-9}$. We have chosen three different mass ratios corresponding to known planetary ring-satellite systems of 

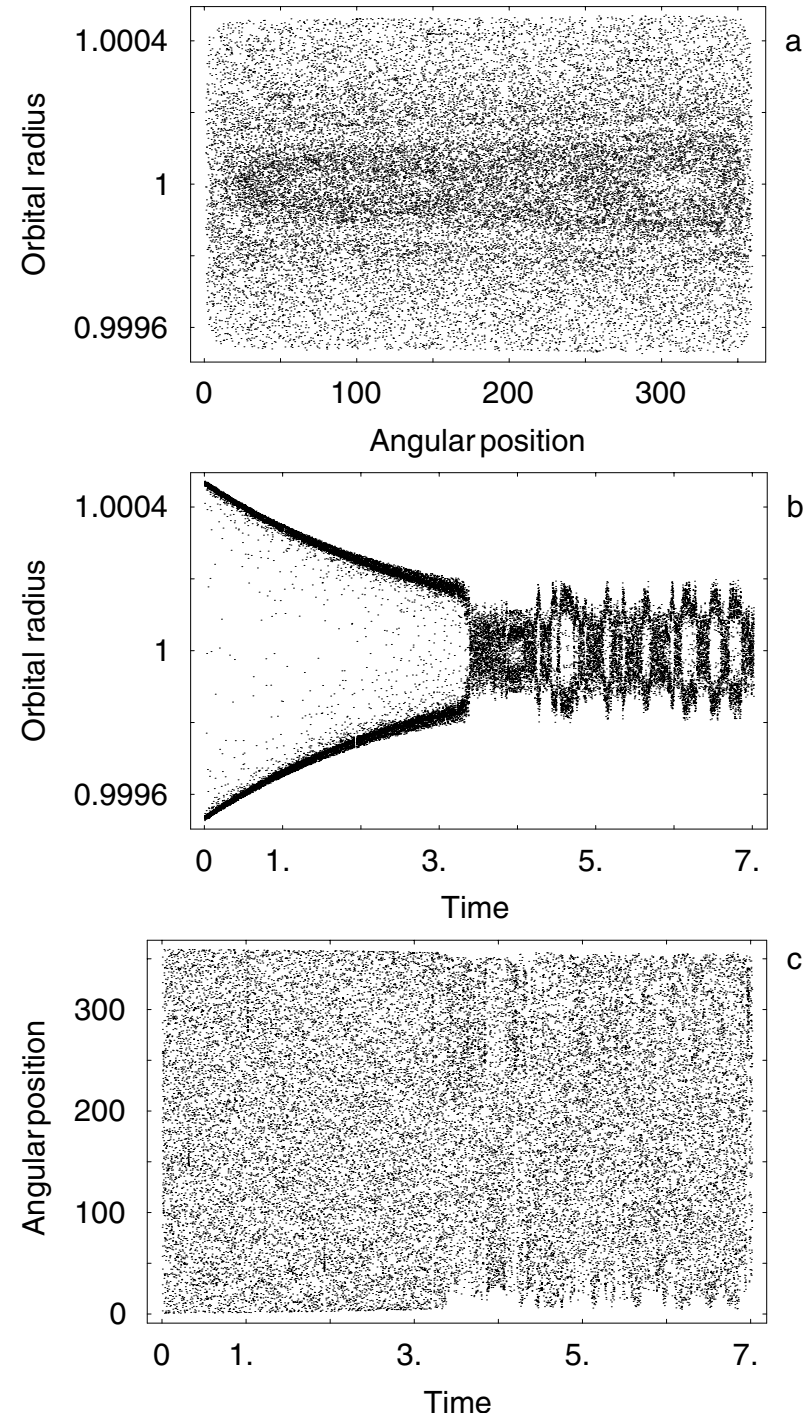

Fig. 2. Orbital evolution of a $1.0 \mu \mathrm{m}$ sized particle under the PRDrag effects and the perturbation of a satellite with mass ratio $\mu_{2}=10^{-9}$. Plots a) and b) show the radial position of the particle as a function of angular position $\theta$ (degrees) and time $\left(\times 10^{5}\right.$ years $)$, respectively. Plot c) shows the variation of $\theta$ as a function of time $\left(\times 10^{5}\right.$ years). The particle starts in a horseshoe orbit and after about $3.3 \times 10^{5} \mathrm{yr}$ shows a highly chaotic behaviour.

Saturn. The $\mu_{2}=10^{-11}$ corresponds to a satellite mass of the order of Pan. We have also analysed the limiting case, $\mu_{2}=10^{-9}$, which corresponds to a satellite mass of the order of Prometheus, the inner satellite of the F ring, and a satellite with $\mu_{2}=10^{-10}$. The sizes of the particles were chosen to be $0.5 \mu \mathrm{m}, 1.0 \mu \mathrm{m}$, and $5.0 \mu \mathrm{m}$. This sample of dust particles can be present in some narrow rings, as has been proposed by Showalter et al. (1992) in the case of the F ring of Saturn.

The results found in the numerical simulations show a different scenario from that proposed in the confinement model. A typical behaviour of the orbital evolution of a particle coorbital to a small satellite under PRDrag perturbation is given in Fig. 2. The evolution can be easily divided into two distinct stages. In the first stage a secular decrease of the radial and the angular amplitudes of oscillation occur. The particle evolves
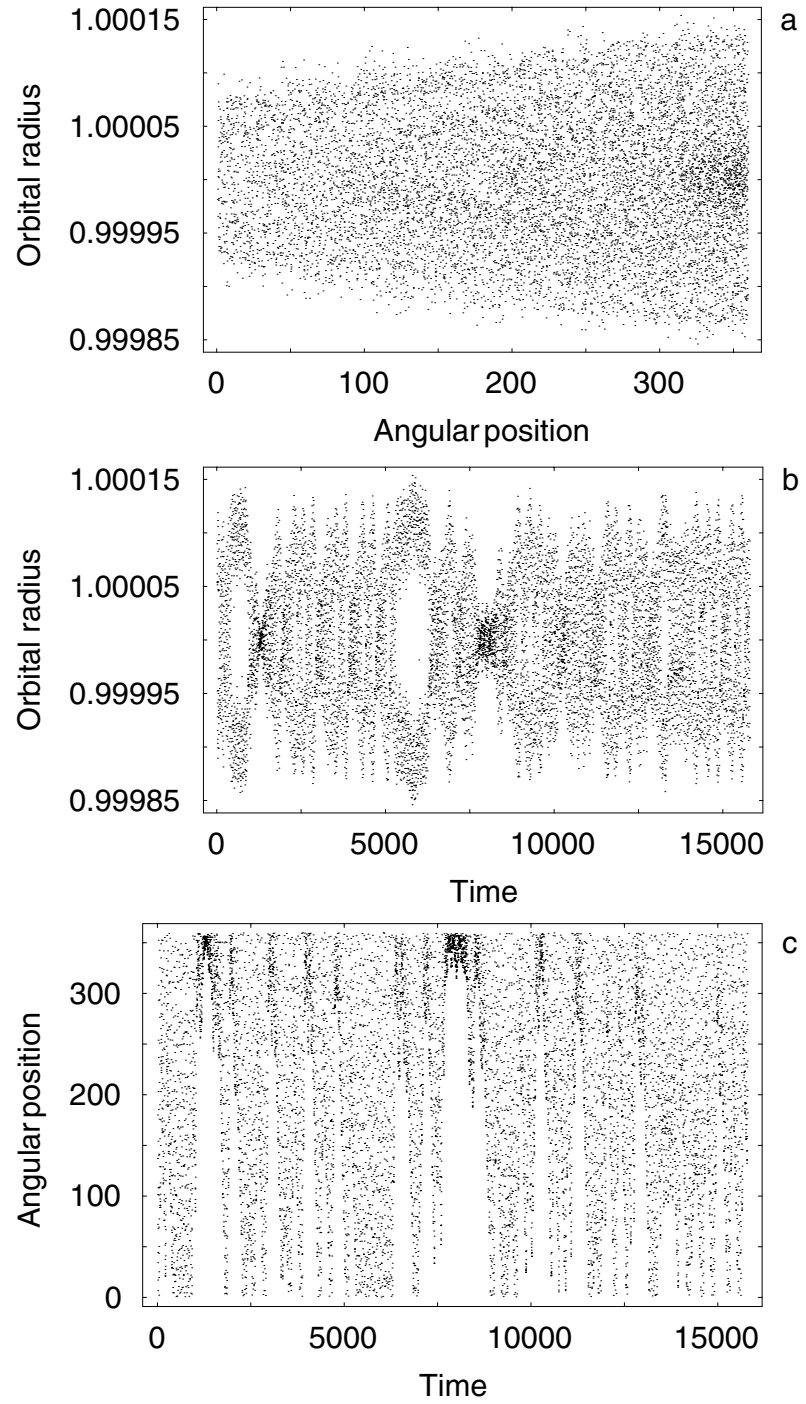

Fig. 3. Orbital evolution of a $0.5 \mu \mathrm{m}$ sized particle under the PRDrag effects and the perturbation of a satellite with mass ratio $\mu_{2}=10^{-10}$. Plots a) and b) show the radial position of the particle as a function of angular position $\theta$ (degrees) and time (years), respectively. Plot c) shows the variation of $\theta$ as a function of time. The particle's motion is highly chaotic, changing its radial and angular oscillation amplitudes randomly.

regularly in horseshoe orbits that keep getting smaller and smaller as time goes on. In the second stage the trajectory evolves in a chaotic way. The radial and angular amplitudes of oscillation evolve erratically, but bounded by the smallest amplitudes of the first stage. There is a critical radial amplitude of oscillation below which the orbital evolution is chaotic.

The critical radial amplitude of oscillation varies as a function of the the particle size and mass ratio. For smaller particle sizes and/or smaller mass ratios the critical radial amplitude of oscillation is larger. For particles with initial radial amplitude of oscillation larger than the critical value the orbital evolution is almost independent of the initial angular position.

The orbital evolution is chaotic in the case where the initial radial amplitude of oscillation is smaller than the critical value (Fig. 3), the first stage does not occur. 

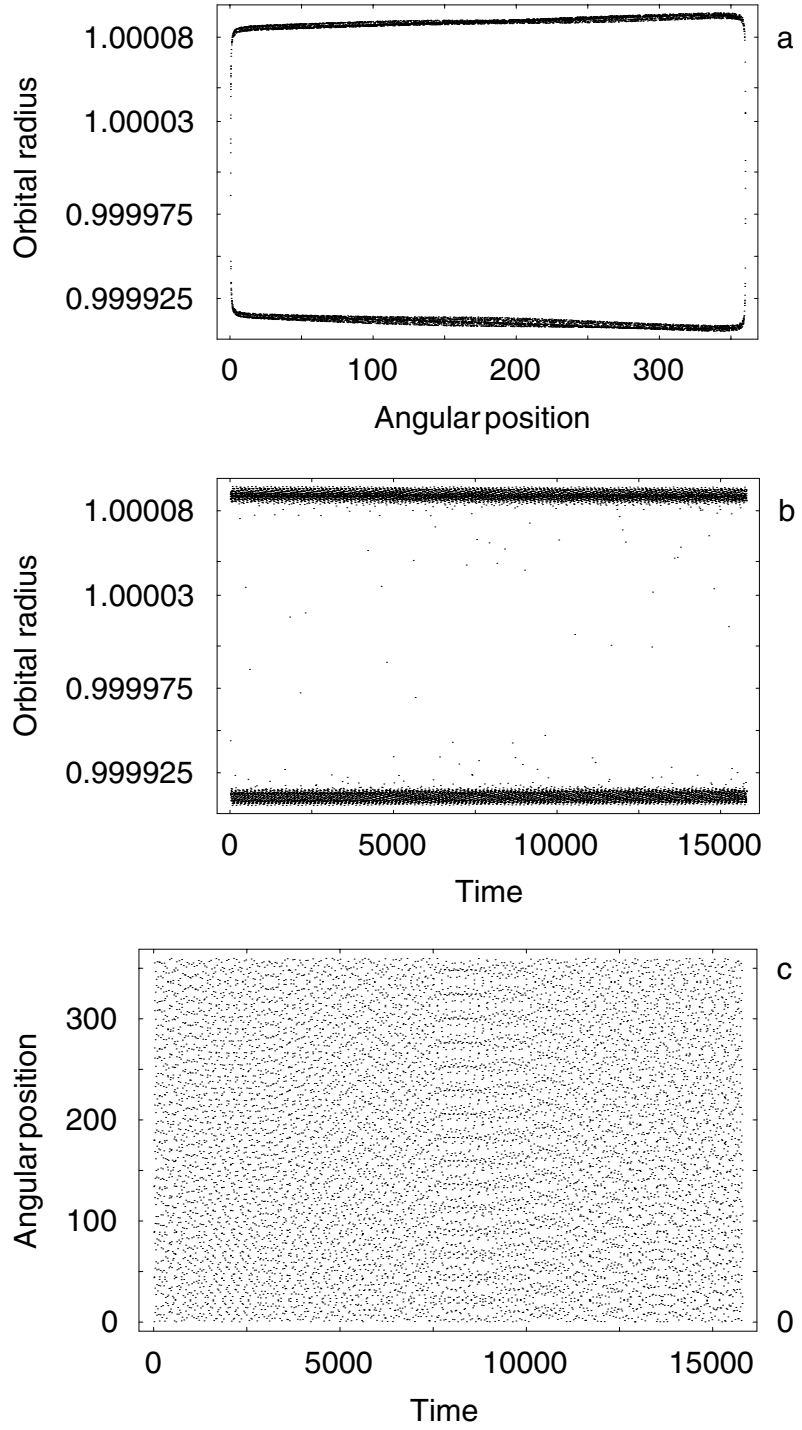

Fig. 4. Orbital evolution of a $5.0 \mu \mathrm{m}$ sized particle under the PRdrag effects and the perturbation of a satellite with mass ratio $\mu_{2}=10^{-10}$. Plots a) and b) show the radial position of the particle as a function of angular position $\theta$ (degrees) and time (years), respectively. Plot c) shows the variation of $\theta$ as a function of time. The particle starts in a horseshoe orbit and apparently keeps its symmetrical path as proposed by Dermott et al. (1979) (see Fig. 1).

Therefore, the symmetry suggested by Dermott et al. (1979, $1980)$ in their ring confinement model does not hold for micrometer-size particles. For particles not too small $(s \geq$ $5.0 \mu \mathrm{m})$ the radial oscillation-amplitude decay is slow, so such that for time scales that are not too long ( $\geq 10^{5}$ years) their orbital evolution in the rotating frame looks as in the model proposed by Dermott (see an example in Fig. 4).

The confinement model as it has been proposed does not include the component relative to the RP since it does not cause a secular variation in the semi-major axis of the particle in contrast to the PRdrag that causes its orbital decay (Burns et al. 1979). For a more general and realistic treatment of this problem we numerically simulated a sample of dust particles under the same conditions of the previous numerical simulations just
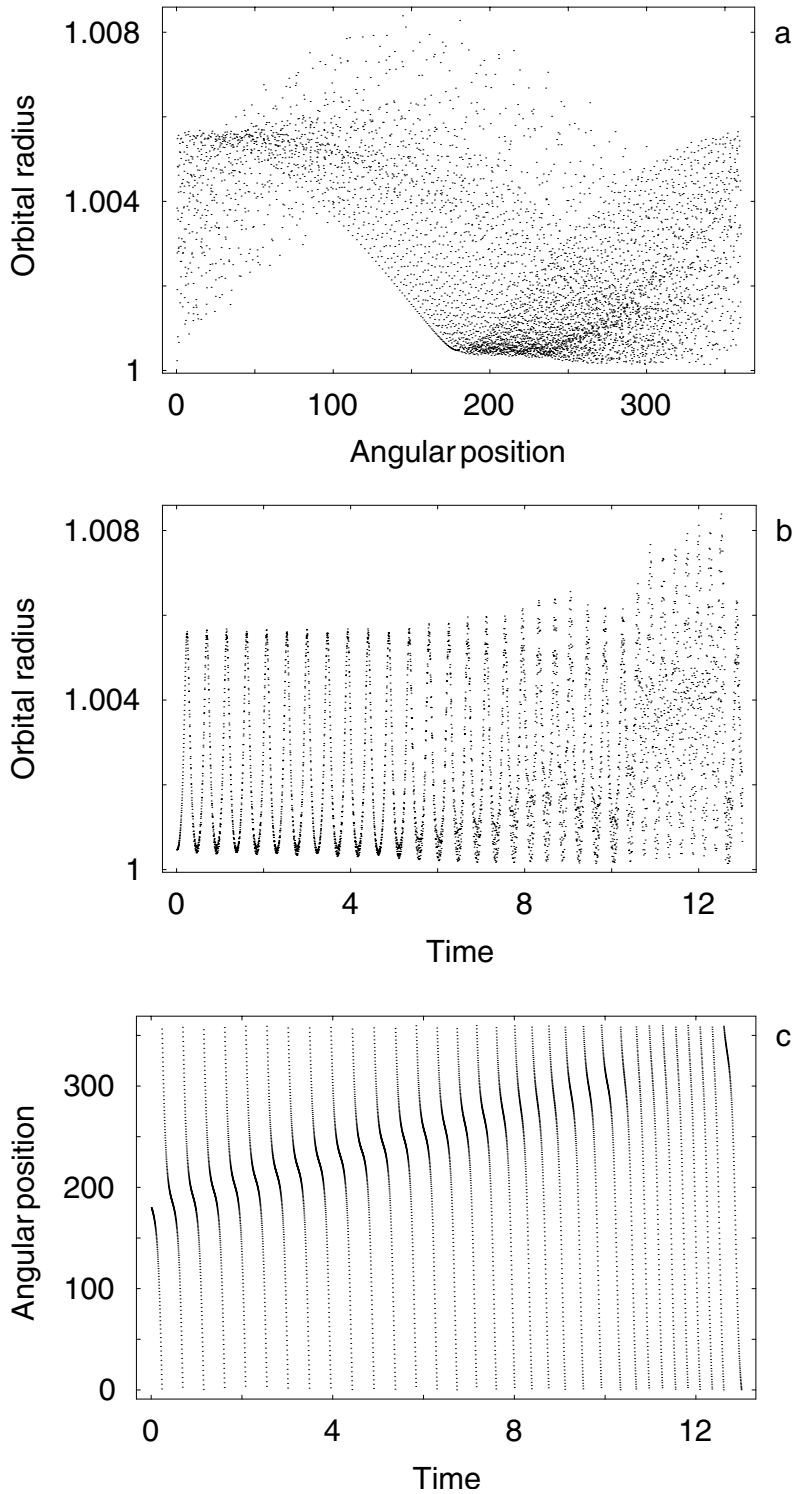

Fig. 5. Orbital evolution of a $1.0 \mu \mathrm{m}$ sized particle under the PRDrag and RP effects and the perturbation of a satellite with mass ratio $\mu_{2}=10^{-9}$. Plots a) and b) show the radial position of the particle as a function of angular position $\theta$ (degrees) and time $\left(\times 10^{5}\right.$ years $)$, respectively. Plot $\mathbf{c}$ ) shows the variation of $\theta$ as a function of time $\left(\times 10^{5}\right.$ years $)$. The particle starts with the same initial conditions as those in Fig. 2, and after about $12 \mathrm{yr}$ it collides with the satellite.

adding the effects of the RP. Our results show that this confinement model is not valid for particles whitin the proposed size ranges of the size of the ring particles and the satellites. The orbit of the particle horseshoeing the satellite will be severely affected and the coorbital configuration will be lost completely. Some particles stay in orbit around the planet away from the derived width of the horseshoe region. Figures 5-7 show the evolution of the particle with the same initial conditions of Figs. 2-4, respectively, but with the inclusion of the radiation pressure component in the system. As we can see, the particles collide within the satellite in a very short time, less than $100 \mathrm{yr}$. 

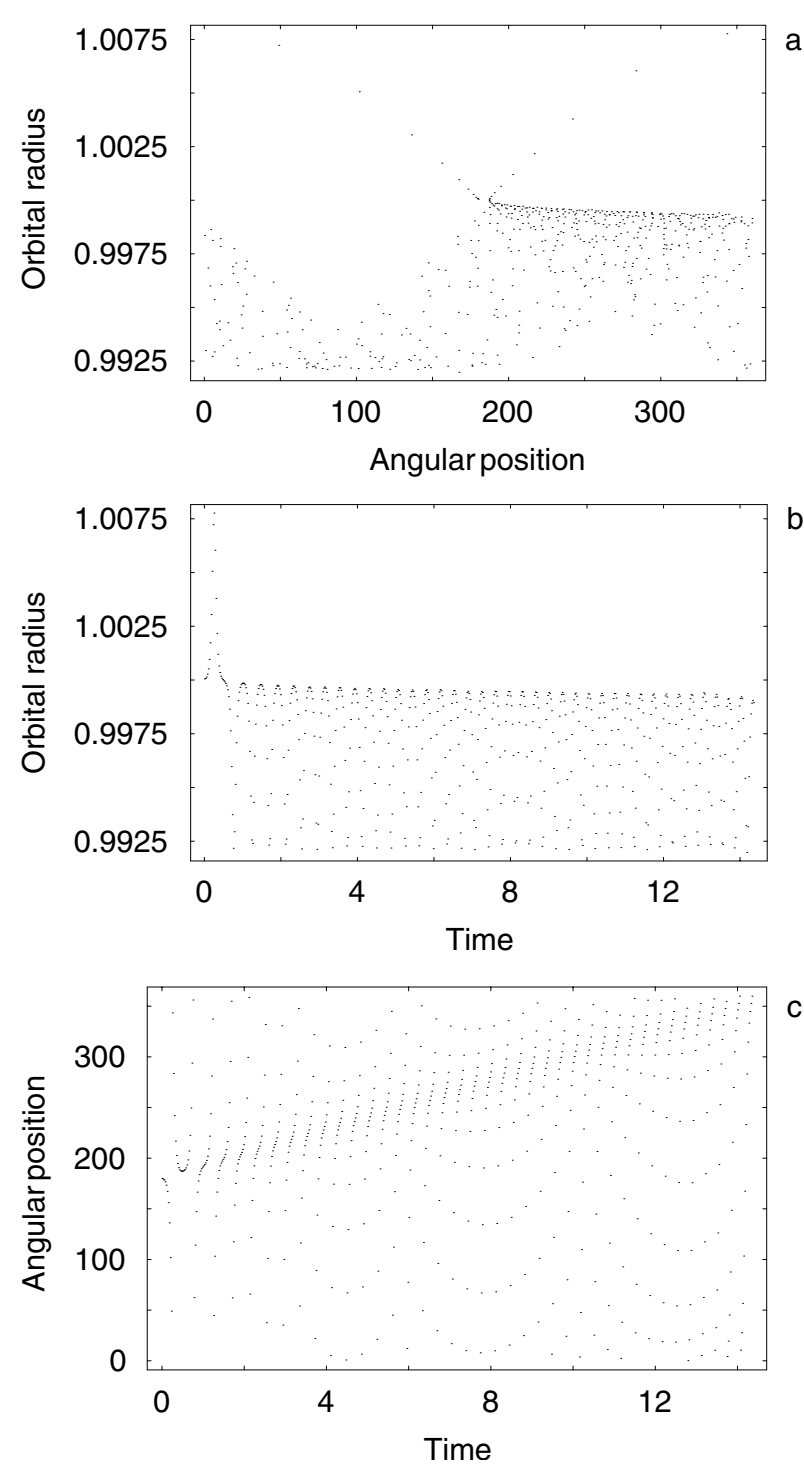

Fig. 6. Orbital evolution of a $0.5 \mu \mathrm{m}$ sized particle under the PRDrag and the RP effects and the perturbation of a satellite with mass ratio $\mu_{2}=10^{-10}$. Plots $\mathbf{a}$ ) and $\mathbf{b}$ ) show the radial position of the particle as a function of angular position $\theta$ (degrees) and time (years), respectively. Plot c) shows the variation of $\theta$ as a function of time. The particle starts with the same initial conditions as in Fig. 3, and after about $14 \mathrm{yr}$ it collides with the satellite.

\section{Final comments}

Since the Poynting-Robertson drag is thought to be the main mechanism responsible for the decay of the dust particles Dermott et al. (1979) have proposed that a satellite can confine a ring particle in horseshoe orbit even in the presence of this dissipative force. In the present work we have tested this confinement model for a range of small particles and satellites.

In all the numerical simulation the particles, under the effects of the PRdrag and the gravitational influence of the satellite, remain confined in the horseshoe region. However, their orbital evolution in the rotating frame is much more complex than the symmetrical path formerly suggested. In general, the particles present two stages of orbital evolution. In the first stage
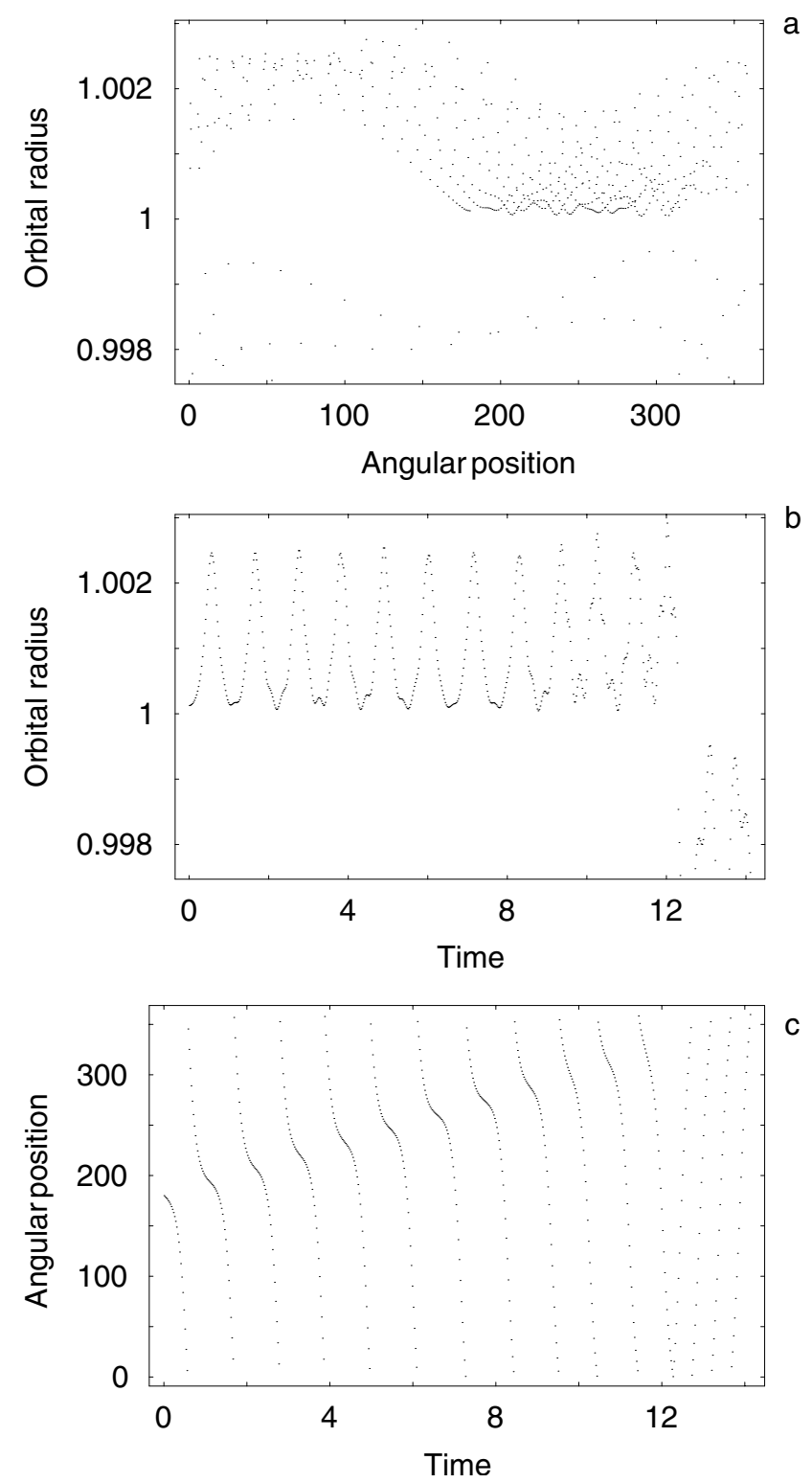

Fig. 7. Orbital evolution of a $5.0 \mu \mathrm{m}$ sized particle under the PRDrag and the RP effects and the perturbation of a satellite with mass ratio $\mu_{2}=10^{-10}$. Plots $\mathbf{a}$ ) and $\mathbf{b}$ ) show the radial position of the particle as a function of angular position $\theta$ (degrees) and time (years), respectively. Plot c) shows the variation of $\theta$ as a function of time. The particle starts with the same initial conditions as in Fig. 4, and after about $14 \mathrm{yr}$ it collides with the satellite.

there is a regular decrease of radial and angular amplitudes of oscillation. In the second stage the particles evolve chaotically with random amplitudes of oscillation.

However, since the radiation-pressure component is also present in the system, we have also included it in the numerical simulations. Although its effect does not cause a secular variation in the semi-major axis, the combined effects destroy the confinement mechanism for dust particles within the range of values mentioned in Sect. 3. Therefore, the timescale proposed for the decaying of dust ring particles is much shorter than the value expected from the confinement model. 
Acknowledgements. We would like to thank the anonymous referee for the helpful review. This work was funded by Fapesp under the Grants 99/11965-8 and 99/11744-1, CNPq and Fundunesp. These supports are gratefully acknowledged.

\section{References}

Burns, J., Lamy, P., \& Soter, S. 1979, Icarus, 40, 1 Dermott, S. F., Gold, T., \& Sinclair, A. T. 1979, AJ , 84, 8

Dermott, S. F., Murray, C. D., \& Sinclair, A. T. 1980, Nature, 248, 309 Dermott, S. F., \& Murray, C. D. 1981, Icarus, 48, 1

Dermott, S. F. 1984, in Planetary Rings (Tucson: University of Arizona Press), 589
Everhard, E. 1985, in Dynamics of Comets: their origin and evolution (The Netherlands: Reidel Publishing Company), 185

Goldreich, P., \& Tremaine, S. 1979, Nature, 277, 97

Mignard, F. 1984, in Planetary Rings (Tucson: University of Arizona Press), 333

Murray, C. D., \& Thompson, R. 1990, Nature, 348, 499

Murray, C. D., \& Dermott, S. F. 1999, Solar System Dynamics (Cambridge: Cambridge Univ. Press)

Porco, C., \& Goldreich, P. 1987, AJ, 93, 724

Showalter, M. R., Pollack, J. B., Ockert, M. E., et al. 1992, Icarus, 100,394

Showalter, M. R. 1991, Nature, 351, 709

Schuerman, D. W. 1979, ApJ, 238, 337 